\title{
Natural Quintessence in String Theory
}

\author{
Michele Cicoli, ${ }^{a, b}$ Francisco G. Pedro, ${ }^{c}$ Gianmassimo Tasinato ${ }^{d}$ \\ ${ }^{a}$ Abdus Salam ICTP, Strada Costiera 11, Trieste 34014, Italy \\ ${ }^{b}$ INFN, Sezione di Trieste, Italy \\ ${ }^{c}$ Rudolf Peierls Centre for Theoretical Physics, University of Oxford, \\ 1 Keble Road, Oxford, OX1 3NP, UK \\ ${ }^{d}$ Institute of Cosmology \& Gravitation, University of Portsmouth, \\ Dennis Sciama Building, Portsmouth, PO1 3FX, United Kingdom \\ E-mail: mcicoli@ictp.it,f.pedro1@physics.ox.ac.uk, \\ gianmassimo.tasinato@port.ac.uk
}

\begin{abstract}
We introduce a natural model of quintessence in string theory where the light rolling scalar is radiatively stable and couples to Standard Model matter with weaker-thanPlanckian strength. The model is embedded in an anisotropic type IIB compactification with two exponentially large extra dimensions and $\mathrm{TeV}$-scale gravity. The bulk turns out to be nearly supersymmetric since the scale of the gravitino mass is of the order of the observed value of the cosmological constant. The quintessence field is a modulus parameterising the size of an internal four-cycle which naturally develops a potential of the order (gravitino mass $)^{4}$, leading to a small dark energy scale without tunings. The mass of the quintessence field is also radiatively stable since it is protected by supersymmetry in the bulk. Moreover, this light scalar couples to ordinary matter via its mixing with the volume mode. Due to the fact that the quintessence field is a flat direction at leading order, this mixing is very small, resulting in a suppressed coupling to Standard Model particles which avoids stringent fifth-force constraints. On the other hand, if dark matter is realised in terms of Kaluza-Klein states, unsuppressed couplings between dark energy and dark matter can emerge, leading to a scenario of coupled quintessence within string theory. We study the dynamics of quintessence in our set-up, showing that its main features make it compatible with observations.
\end{abstract}




\section{Contents}

1 Introduction 1

2 Natural quintessence from anisotropic compactifications $\quad 3$

2.1 Naturally light quintessence field 3

2.2 Naturally decoupled quintessence field 5

$\begin{array}{lll}3 & \text { Explicit form of the quintessence potential } & 8\end{array}$

4 Quintessence coupling to matter $\quad 12$

4.1 Coupling to gauge bosons 12

4.2 Coupling to fermions 13

5 Dynamics of the quintessence-dark matter system $\quad 14$

5.1 Decoupled quintessence and dark matter $\quad 15$

$\begin{array}{lll}5.2 & \text { Coupled quintessence and dark matter } & 17\end{array}$

6 Conclusions 20

$\begin{array}{ll}\text { A Canonical normalisation } & 21\end{array}$

B Explicit form of the coefficients of the mass matrix 23

\section{Introduction}

Present cosmological observations indicate that our Universe is currently accelerating. This accelerated expansion is driven by a mysterious form of energy density with negative pressure, dubbed 'dark energy', that accounts for about $70 \%$ of the total energy density of the Universe. The major part of the remaining 30\% consists of cold non-baryonic dark matter, whose microscopic origin is not known, although it is likely to be associated with new particles as supersymmetric partners, axions, string moduli or hidden sector degrees of freedom. Finally, ordinary baryonic matter accounts for only a few\% of the total energy density.

The microscopic origin of dark energy is even less clear than that of dark matter. Many proposals have been pushed forward for explaining present day acceleration (see [1] for a comprehensive review). We will focus here on quintessence models, in which the dynamics of scalar fields drives the present cosmological acceleration, as opposed to the simplest scenario in which the cosmological energy density is dominated today by a pure cosmological constant.

Both of these proposals, a rolling quintessence field and a non-zero cosmological constant, have their own virtues and shortcomings:

- Cosmological Constant Problem: If dark energy is given by a non-zero cosmological constant, in order to match the current cosmological observations, its value needs to be of the order $\langle V\rangle=\Lambda^{4} \sim \mathrm{meV}^{4} \sim 10^{-120} M_{P}^{4}$. Whereas, if dark energy is provided by a quintessence model, in which a late-time acceleration is characterised by a scalar $\phi$ with kinetic energy smaller than its potential energy, the corresponding scalar mass 
has to be of the order $m \sim 10^{-33} \mathrm{eV}$. The two values of $\Lambda$ and $m$ are related to each other since:

$$
m^{2} \sim V_{\phi \phi} \sim \frac{V}{\phi^{2}} \lesssim \frac{V}{M_{P}^{2}} \sim 10^{-120} M_{P}^{2} \sim\left(10^{-33} \mathrm{eV}\right)^{2} .
$$

The condition $\phi \gtrsim M_{P}$ follows from the Friedmann equation $3 M_{P}^{2} H^{2}=\left(\dot{\phi}^{2} / 2+V\right) \simeq V$ and the equation of motion for $\phi, 0=\ddot{\phi}+3 H \dot{\phi}+V_{\phi} \simeq 3 H \dot{\phi}+V_{\phi}$. In fact, for $\dot{\phi}^{2} \lesssim V$, we have:

$$
\phi \sim \frac{V}{V_{\phi}} \sim \frac{V}{H \dot{\phi}} \gtrsim M_{P} .
$$

The required smallness of these quantities ( $\Lambda$ or $m$, depending on the scenario under consideration) represents one of the biggest mysteries of modern physics since a naïve estimate of the vacuum energy would yield a result many orders of magnitude larger than the observed one: $\langle V\rangle \sim M_{P}^{4}$ for non-supersymmetric theories and $\langle V\rangle \sim \mathrm{TeV}^{4} \sim$ $10^{-60} M_{P}^{4}$ for theories with TeV-scale supersymmetry. In the quintessence case, one can assume that the vacuum energy is exactly zero (as forced by symmetry arguments, for example), but one should still explain how to obtain such a small mass for the rolling scalar. Indeed, scalar masses are notoriously hard to keep from getting large contributions when integrating out ultra-violet physics running in the loops. This is the issue of technical naturalness, which plays a crucial rôle in the determination of the Higgs mass and the solution of the famous $\eta$-problem in slow-roll inflation. However, here the problem is more severe than in inflation since one has to integrate out all the modes down to the tiny cosmological constant scale $\Lambda \sim \mathcal{O}(\mathrm{meV})$, instead of the inflationary scale $M_{\mathrm{inf}} \sim \mathcal{O}\left(M_{\mathrm{GUT}}\right)$.

- Coincidence Problem: Observations indicate that the energy densities of dark matter and dark energy are of the same order of magnitude at present cosmological epochs. The reason why different forms of matter have similar properties today is a puzzle which goes under the name of 'coincidence problem'. Dark energy models based on a non-zero, pure cosmological constant have little to say about it, but quintessence models can in principle provide answers to this problem. The idea is to consider 'tracking' solutions, in which the non-trivial dynamics of the scalar field leads its energy density to mimic radiation during the radiation-dominated era. The transition between radiation to matter domination, then, triggers quintessence towards assuming characteristics that match the observed behavior of dark energy.

- Fifth-force constraints: Despite providing a potential understanding of the present ratios of energy densities via tracking solutions, quintessence models are generically plagued by the problem of long-range unobserved fifth-forces which would be mediated by such a light scalar field [2]. The present mass bounds for scalars with Planck strength couplings to matter are $m \gtrsim \mathcal{O}(\mathrm{meV})[3]$. Furthermore, a rolling scalar would lead to a time variation of the constants of Nature, while these do not appear to drastically change between Big Bang Nucleosynthesis (BBN) and the present epoch. The standard solution to this problem involves pseudo Goldstone bosons with shift symmetries [4] since these pseudoscalars give rise to spin-dependent couplings which cannot be probed in fifth-force experiments that are insensitive to the polarization. Moreover, the shift symmetry forbids higher dimensional operators, rendering the quintessence field stable against radiative corrections from UV physics. On the other hand, it is much harder to satisfy the current constraints in models where the quintessence field is a scalar. 
Most of the attempts to realise quintessence within string theory rely on axions and shift symmetries [5-8]. In this paper we shall, however, take a different approach, and build the first string model where the quintessence field is a scalar parameterising the size of an internal four-cycle. We shall focus on LARGE Volume Scenarios (LVS) which emerge naturally in type IIB flux compactifications. The set-up of this model represents an interesting variation of the one derived in [9] for inflationary purposes. In fact, as in [9], we focus on the recent string embedding [10] of the Supersymmetric Large Extra Dimension (SLED) proposal [11]. The field which was playing the rôle of the inflaton in [9] will now behaves as quintessence.

In our new model, quintessence is driven by a closed string modulus with a naturally small mass $m$ protected against loop corrections. The mechanism for obtaining such a light scalar whose mass is radiative stable is similar to the one of [12] and we shall outline it in section 2. Contrary to the model of [12] where the quintessence field was the overall volume mode, in our case quintessence is driven by a fibre modulus that at leading order neither develops a potential nor couples to Standard Model (SM) matter. The potential for the quintessence field arises only at subleading order by means of non-perturbative and string loop effects, that also induce suppressed, weaker-than-Planckian couplings with ordinary matter. Thus, our model is able to avoid fifth-force constraints. An intuitive explanation for this non-standard behaviour for the quintessence scalar will be given in section 2 , while in sections 3 and 4 we will develop our arguments at a more technical level.

The dynamics of the quintessence-dark matter system will then be analysed in section 5 starting from the standard decoupled case and then moving to the more complicated coupled case where we shall also explain how our stringy set-up can allow for unsuppressed couplings of the quintessence field with dark matter degrees of freedom. Hence we will suggest a realisation of a coupled quintessence-dark matter model inspired by string theory. Finally we will conclude in section 6 .

\section{Natural quintessence from anisotropic compactifications}

In this section, we shall outline in some detail the ideas behind the construction of our quintessence model, before developing the technical elements of our set-up in the next sections.

\subsection{Naturally light quintessence field}

In our approach, a naturally small mass for the quintessence field is generated by means of a string realisation of the so-called Supersymmetric Large Extra Dimension (SLED) scenario (see [13] for a review). In its original form, this is a brane-world model with two large extra dimensions, in which supersymmetry is preserved in the bulk, whereas it is only nonlinearly realised on a Standard Model codimension-two brane. Its main attractive feature is the possibility to tie the discussion of the hierarchy problem to that of the cosmological constant problem. More precisely, the hierarchy problem is addressed by the presence of large extra dimensions which lower the fundamental gravity scale down to the TeV-scale; at the same time, the observed value of the cosmological constant is associated with the low supersymmetry breaking scale in the bulk. These two arguments are tied up since simple considerations based on dimensional reduction yield the following relation among the fundamental $(4+d)$-dimensional gravity scale $M_{*}$ and the gravitino mass $m_{3 / 2}$ :

$$
m_{3 / 2} \sim\left(\frac{M_{*}}{M_{P}}\right)^{2} M_{P}
$$


Setting $M_{*} \sim \mathcal{O}(\mathrm{TeV}) \sim 10^{-15} M_{P}$, the gravitino mass turns out to be very small, of the order of the dark energy scale $m_{3 / 2} \sim \Lambda^{1 / 4} \sim \mathcal{O}(\mathrm{meV}) \sim 10^{-30} M_{P}$. This implies that the bulk is nearly supersymmetric, given that supersymmetry is broken only at the dark energy scale. Quantum corrections to the vacuum energy from loops of bulk fields scale as:

$$
V_{\text {loop }} \sim \operatorname{Str}\left(M^{2}\right) \Lambda_{\mathrm{UV}}^{2} \sim m_{3 / 2}^{2} M_{\mathrm{KK}}^{2},
$$

where $\operatorname{Str}\left(M^{2}\right) \sim m_{3 / 2}^{2}$ and the cut-off is taken to be the scale at which a pure 4D description ceases to be valid: $\Lambda_{\mathrm{UV}} \sim M_{\mathrm{KK}}$. Given that the Kaluza-Klein scale is given by:

$$
M_{\mathrm{KK}} \sim\left(\frac{m_{3 / 2}}{M_{P}}\right)^{1 / 2+1 / d} M_{P}
$$

this is of the same order of $m_{3 / 2}$ for $d=2$. This is also the case that for $\mathrm{TeV}$-scale fundamental gravity leads to micron-sized extra dimensions which are at the edge of detectability in experiments that look for deviations from Newton's law. Hence in the case of two large extra dimensions, the loop generated potential (2.2) gives the correct observed order of magnitude of the cosmological constant since it scales as $V_{\text {loop }} \sim m_{3 / 2}^{4} \sim 10^{-120} M_{P}$. Notice that for energies above the cut-off $M_{\mathrm{KK}}$, the theory is effectively $6 \mathrm{D}$ and supersymmetric, and so the contributions to the vacuum energy from loops of bosons and fermions cancel among each other.

In order to have a viable and complete solution of the cosmological constant problem, one has to make sure that (2.2) is the leading order contribution to the vacuum energy. In general, one would expect larger contributions coming directly from the tension of the Standard Model brane, of the order $T \sim \mathcal{O}(\mathrm{TeV})^{4}$, and from the tree-level part of the bulk potential corresponding to the curvature of the extra dimensions induced by the presence of the $\mathrm{TeV}$ brane. This is a delicate issue (see e.g. [14]): recent progresses seem to suggest that consistent scenarios can be built along these lines by means of suitable couplings between bulk fields and the SM brane [15]. Since the rest of our discussion does not rely specifically on these details, we will not develop this issue any further in this paper.

The SLED framework opens the possibility to obtain a natural model of quintessence. In fact, in 6D SLED models, the radion mode $r$ develops a potential via loops of bulk fields (Casimir energy) which scale as (2.2), and so give it a mass of the order [12]:

$$
m_{r}^{2} \sim \frac{V_{\text {loop }}}{M_{P}^{2}} \sim \frac{m_{3 / 2}^{4}}{M_{P}^{2}} \sim\left(10^{-33} \mathrm{eV}\right)^{2} .
$$

This is the perfect scale for quintessence, since it is the typical mass scale expected for a quintessential scalar; for the arguments we outlined above, this small mass is technically natural since it is radiatively stable thanks to the unbroken supersymmetry in the bulk (see [12] for more details).

In the quintessence model based on the $6 \mathrm{D}$ SLED scenario, the radion mode, being a scalar instead of a pseudo-scalar with Planck strength coupling to ordinary matter, would mediate long range fifth-forces. Moreover its cosmological evolution would lead to a time dependence of Newtons constant. The authors of [12] propose to cure this problem exploiting the fact that the couplings of the radion to matter are field dependent, approaching small values for appropriate cosmological evolutions of the radion field. The strongest constraint comes from the requirement that the Newton's constant does not change that much from BBN till today. 
We will now argue that a natural, automatic solution to the fifth-force problem emerges when embedding this quintessence scenario in string theory. A recent paper [10] discusses how to embed these 6D SLED scenarios within type IIB flux compactifications. The simplest Calabi-Yau three-fold that allows such a derivation has a fibred structure with a 4D K3 or $T^{4}$ fibre over a $2 \mathrm{D} \mathbb{P}^{1}$ base. This structure allows to take a very anisotropic limit where the base is exponentially larger than fibre, resulting in an intermediate $6 \mathrm{D}$ effective field theory when compactifying 10D type IIB string theory down to $4 \mathrm{D}$. The authors of [10] show how to stabilise the geometric moduli in such a way to obtain the above mentioned anisotropic limit within the framework of the LARGE Volume Scenario (LVS) [17]. In this framework, exponentially large extra dimensions emerge from a moduli stabilisation mechanism that exploits a combination of non-perturbative and $\alpha^{\prime}$ effects. This set-up provides the perfect arena for building a SLED-motivated quintessential scenario in IIB string theory: in the next subsection we will outline why and how it automatically solves the problem of fifth-forces.

\section{$2.2 \quad$ Naturally decoupled quintessence field}

The explicit string embedding reveals that the $6 \mathrm{D}$ effective field theory is far more richer than the one considered in [12], due to the presence of additional closed string moduli on top of the radion (that in the IIB embedding corresponds to the volume mode). Moreover, besides the equivalent of the loop corrections (2.2), both higher-derivative contributions $\left(\alpha^{\prime}\right.$ corrections) and non-perturbative effects play a crucial rôle in determining the bulk action. These additional features of the $6 \mathrm{D}$ effective field theory, obtained as the low-energy limit of an anisotropic fibred compactification, might at first glance seem a source of new complications for getting a viable quintessence model. However, a deeper analysis reveals that the string embedding actually provides an elegant quintessence model, with a natural solution to the fifth-force problem.

The minimal scenario we consider in this work involves three Kähler moduli, which parameterise the size in string units of internal four-cycles, and a fibered Calabi-Yau volume of the form [16]:

$$
\mathcal{V}=t_{b} \tau_{f}-\tau_{s}^{3 / 2} .
$$

The fields defining the volume have the following rôles and geometrical interpretation:

- $\tau_{s}$ is a del Pezzo divisor supporting non-perturbative effects of the form $W_{\mathrm{np}}=A e^{-a T_{s}}$, where $\tau_{s}=\operatorname{Re}\left(T_{s}\right)$ and $A$ and $a$ are $\tau_{s}$-independent constants. These effects fix $\tau_{s}$ at 'small' values but still in a geometric regime above the string scale: $\left\langle\tau_{s}\right\rangle \sim \mathcal{O}(10)$.

- The overall volume mode $\mathcal{V} \simeq t_{b} \tau_{f}$ is a modulus fixed at exponentially large values, $\langle\mathcal{V}\rangle \sim e^{1 / g_{s}} \gg 1$ for weak string coupling $g_{s} \lesssim 0.1$, by the interplay of non-perturbative effects and $\alpha^{\prime}$ corrections to the Kähler potential [17]. The size of the $2 \mathrm{D} \mathbb{P}^{1}$ base is given by $t_{b}$ whereas the volume of the $4 \mathrm{D} \mathrm{K} 3$ or $T^{4}$ fibre is given by $\tau_{f}$. The value of $\mathcal{V}$ plays a crucial rôle in determining the scales and coupling strengths of our model, explaining in a natural way why the size of dark energy is so small.

- The fibre modulus $\tau_{f}$ plays the rôle of the quintessence field. It develops a potential only a subleading order due to poly-instanton corrections to the superpotential generated by an E3-instanton wrapping this non-rigid divisor: $W_{\mathrm{np}}=A e^{-a\left(T_{s}+C e^{-2 \pi T_{f}}\right)}$, where again $\tau_{f}=\operatorname{Re}\left(T_{f}\right)$ and $C$ is a $\tau_{f}$-independent constant. These tiny effects fix the fibre modulus 'small', i.e. at the same order of magnitude of $\tau_{s}:\left\langle\tau_{f}\right\rangle \sim\left\langle\tau_{s}\right\rangle$. This gives rise to a very anisotropic compactification with $t_{b}$ exponentially larger than $\sqrt{\tau_{f}}$. 
None of these three four-cycles can support the visible sector where supersymmetry is nonlinearly realised via a proper configuration of branes wrapped around internal divisors. ${ }^{1}$ In fact, the Standard Model cannot be localised neither on $\tau_{s}$ nor on $\tau_{f}$ due to the tension between chirality and the generation of non-perturbative effects [18]. Moreover, the four-cycle containing the base, $\tau_{b} \simeq t_{b} \sqrt{\tau_{f}}$, would give rise to an exponentially small gauge coupling $g^{-2} \simeq \tau_{b} \gg 1$. Thus we need to add a fourth Kähler modulus for the Standard Model, $\tau_{\mathrm{SM}}$. This divisor has to be rigid in order to avoid undesired matter in the adjoint representation and has to be fixed at small values in order to get a correct value of the gauge coupling. This can be done by exploiting D-terms and string loop corrections to the Kähler potential [19]. Therefore $\tau_{\mathrm{SM}}$ has the same features as $\tau_{s}$ apart from the fact that one cycle is fixed perturbatively whereas the other non-perturbatively. Hence, without loss of generality, we shall just restrict ourselves to the simplest case with three moduli having in mind that the modulus $\tau_{s}$ can represent a generic rigid and 'small' divisor which can be either $\tau_{\mathrm{SM}}$ or the one supporting the non-perturbative effects.

The structure of the scalar potential for the bulk fields (trading $t_{b}$ for $\mathcal{V}$ ) is the following:

$$
V=V_{\mathrm{np}}\left(\mathcal{V}, \tau_{s}\right)+V_{\alpha^{\prime}}\left(\mathcal{V}, \tau_{s}\right)+V_{\text {poly }}\left(\mathcal{V}, \tau_{s}, \tau_{f}\right)+V_{\text {loop }}\left(\mathcal{V}, \tau_{s}, \tau_{f}\right)
$$

where the leading order effects are given by the non-perturbative and $\alpha^{\prime}$ potentials, $V_{\mathrm{np}}$ and $V_{\alpha^{\prime}}$, which depend only on $\mathcal{V}$ and $\tau_{s}$ and scale as:

$$
V_{\text {lead }}=V_{\text {np }}\left(\mathcal{V}, \tau_{s}\right)+V_{\alpha^{\prime}}\left(\mathcal{V}, \tau_{s}\right) \sim \mathcal{O}\left(m_{3 / 2}^{3} M_{P}\right)
$$

The previous potential $V_{\text {lead }}$ determines the properties of the volume and the modulus $\tau_{s}$ at leading order in an inverse volume expansion (since the volume is exponentially large, its inverse represents an ideal expansion parameter). This implies that the basic features of SM physics, like gauge and Yukawa couplings, are determined at leading order by the VEV of $\tau_{s}$. Instead, in our string construction, the fibre modulus $\tau_{f}$ develops a potential only at subleading order due to poly-instanton effects and loops of closed strings propagating in the bulk. The subleading potential scales as $(2.2):^{2}$

$$
V_{\text {sub }}=V_{\text {poly }}\left(\mathcal{V}, \tau_{s}, \tau_{f}\right)+V_{\text {loop }}\left(\mathcal{V}, \tau_{s}, \tau_{f}\right) \sim \mathcal{O}\left(m_{3 / 2}^{4}\right)
$$

If the tension of the Standard Model brane is compensated by the brane back-reaction, as in the 6 D SLED scenario, the leading order contribution to the vacuum energy is given by (2.7) which does not reproduce the correct value of the cosmological constant for $m_{3 / 2} \sim \mathcal{O}(\mathrm{meV})$ obtained from (2.1) setting the string scale $M_{s}=M_{*}$ of the order the TeV scale. However the potential (2.7) gives rise to a SUSY breaking AdS vacuum, which still needs to be uplifted to a dS solution. This can be successfully accomplished by means of one of the many mechanisms known in the literature (tension of anti branes at the tip of warped throats [20], D-terms from magnetised branes [21] or dilaton-dependent non-perturbative effects [22]). The leading order contribution to the vacuum energy would then be given by the subleading potential

\footnotetext{
${ }^{1}$ We need to focus on a non-linear realisation of supersymmetry where the low-energy effective field theory contains just the Standard Model without any superpartner since models with TeV-scale strings predict $m_{3 / 2} \sim$ $\mathrm{meV}$, and so gravity mediation would give rise to soft terms of this unacceptably small order of magnitude.

${ }^{2}$ We do not include the contribution coming from loops of open string fields since by construction no D7-brane stack wraps either the fibre or the base divisor. Hence open strings are localised far away in the Calabi-Yau, and so do not develop any potential for $\tau_{1}$. If that were the case, the internal manifold would have a isotropic shape since $\tau_{1}$ would also be fixed at exponentially large values: $\left\langle\tau_{1}\right\rangle \simeq g_{s}^{4 / 3}\langle\mathcal{V}\rangle^{2 / 3} \gg 1$.
} 
(2.8) which now naturally gives rise to the observed size of dark energy, since it scales as the bulk loop potential of 6D SLED models (see (2.2)).

A key difference between our scenarios and SLED constructions is the fact that the volume mode can no longer be used as the quintessence field, since it develops a potential at order $m_{3 / 2}^{3} M_{P}$, and so the corresponding mode is too heavy to drive quintessence:

$$
m_{\mathcal{V}}^{2} \sim \frac{V_{\text {lead }}}{M_{P}^{2}} \sim m_{3 / 2}^{2}\left(\frac{m_{3 / 2}}{M_{P}}\right) \sim\left(10^{-18} \mathrm{eV}\right)^{2} .
$$

On the other hand, the fibre modulus $\tau_{f}$ does not appear in the expression (2.7) for $V_{\text {lead }}$, and so its mass has the perfect quintessential value which can be estimated from the subleading potential (2.8):

$$
m_{\tau_{f}}^{2} \sim \frac{V_{\mathrm{sub}}}{M_{P}^{2}} \sim m_{3 / 2}^{2}\left(\frac{m_{3 / 2}}{M_{P}}\right)^{2} \sim\left(10^{-33} \mathrm{eV}\right)^{2} .
$$

This expression is similar to (2.4) which gives the mass of the radion mode of vanilla $6 \mathrm{D}$ SLED models. Consequently the natural explanation of why the quintessence field is light and radiatively stable is, in practice, the same in both scenarios. However, the radion mode in the SLED proposal couples to ordinary matter with Planck strength, $1 / M_{P}$. Instead, as discussed also in [10], the fibre modulus has a weaker-than-Planckian coupling of the order $\epsilon / M_{P}$ where $\epsilon \sim m_{3 / 2} / M_{P} \ll 1$. For this reason, this set-up is ideal for building a quintessence model since the rolling field can safely evade all the current bounds from fifth-force experiments.

However, given that $\mathcal{V}$ couples to ordinary matter as $1 / M_{P}$, these bounds would not be evaded by the volume mode if it had a mass below the meV scale as estimated in (2.9). Nonetheless, supersymmetry is only non-linearly realised on the SM brane, and so the moduli masses (2.9) and (2.10) might get large radiative corrections from loops of open strings. ${ }^{3}$ As estimated in [10], this is indeed the case for the volume mode whose mass receives a correction of the order:

$$
\delta m_{\mathcal{V}} \simeq \frac{M_{s}^{2}}{M_{P}} \simeq m_{3 / 2} \sim \mathcal{O}(\mathrm{meV}),
$$

which renders this modulus still compatible with fifth-force bounds. We stress that the mass of the quintessence field does not get lifted by loops of brane fields due to the weakness of its coupling. In fact, the correction estimated in [10] scales as:

$$
\delta m_{\tau_{f}} \simeq \epsilon \frac{M_{s}^{2}}{M_{P}} \simeq \epsilon m_{3 / 2} \sim \mathcal{O}\left(10^{-33} \mathrm{eV}\right),
$$

and so does not lift the mass (2.10).

The intuitive reason of the weakness of the fibre modulus coupling to ordinary matter is the fact that the leading order potential $V_{\text {lead }}$ does not depend on $\tau_{f}$ (see (2.7)), and so there is no leading order mixing between $\tau_{f}$ and the volume mode $\mathcal{V}$. The mixing between $\tau_{f}$ and $\mathcal{V}$ arises only at subleading order at the level of $V_{\text {sub }}$ (see (2.8)). Therefore the order of magnitude of this small mixing, $\epsilon \ll 1$, is weighted by the ratio between $V_{\text {sub }}$ and $V_{\text {lead }}$ :

$$
\epsilon \sim \frac{V_{\text {sub }}}{V_{\text {lead }}} \sim \mathcal{O}\left(\frac{m_{3 / 2}}{M_{P}}\right)
$$

\footnotetext{
${ }^{3}$ These loops of brane fields contribute to the brane tension which has to be cancelled by the brane backreaction. However this cancellation concerns just the vacuum energy, i.e. the value of the potential at the minimum.
} 
Given that the volume mode couples to ordinary matter as $1 / M_{P}$ and the coupling of the fibre modulus to SM particles is induced by the mixing between $\tau_{f}$ and $\mathcal{V}$, the coupling of the fibre modulus has to be suppressed with respect to the one of the volume mode by the small mixing parameter $\epsilon$, explaining the weaker-than-Planckian nature of this interaction. In other words, the weakness of this coupling is reflecting the fact that $\tau_{f}$ is a flat direction at leading order and does not couple with ordinary matter at that level. On the other hand, the quintessence field has the opportunity to couple with Planckian strength to massive degrees of freedom (as stable 6D Kaluza-Klein states) that can provide a model of dark matter. Consequently, our set-up can lead to a stringy realisation of an interacting dark energy-dark matter system.

We shall make this analysis more precise in section 4 where we will compute the moduli couplings to Standard Model particles. These are extracted from the moduli dependence of the kinetic and mass terms of gauge bosons and fermions, using the canonical normalisation carried out in appendix A.

After this outline of the properties of our quintessence scenario, we pass to develop the technical details.

\section{Explicit form of the quintessence potential}

In this section, we start developing our set-up by discussing the features of the potential that drives quintessence. As explained in section 2, we work within the framework of the LARGE Volume Scenario of type IIB string theory [17] where the volume is fixed at exponentially large values by combining $\alpha^{\prime}$ corrections to the Kähler potential $K$ and non-perturbative contributions to the superpotential $W$.

As explained in subsection 2.2, we consider compactifications where the volume of the Calabi-Yau manifold has a fibered structure and is given by (2.5). With this choice of internal manifold, the standard LVS framework stabilises only two of the three Kähler moduli. The remaining flat direction, which can be identified with the fibre modulus $\tau_{f}$, is lifted either by string loop corrections to $K[23]$ or by poly-instanton effects $[9,10]$. Here we focus our attention on the latter possibility, and investigate if the resulting potential for the fibre modulus $\tau_{f}$ allows us to provide a string theory realisation of quintessence. The analysis closely follows that of our previous paper [9], in which we studied a similar scalar potential in the context of an inflationary model. For this reason, we will only sketch the derivation and the properties of the scalar potential here, referring the reader to [9] for more details.

It was shown in [24] that there are instantons which do not give rise to a single contribution to the superpotential but, due to the presence of extra fermionic zero-modes, they correct the action of another instanton wrapping a different internal cycle. These poly-instantons generate corrections to the superpotential of the form $W_{\mathrm{np}} \sim e^{-\left(T_{i}+e^{-T_{j}}\right)}$. These double exponential terms were used in [10] to provide a string realisation of 6D SLED scenarios and, in [9], to provide a controlled model of inflation in the Kähler moduli sector.

In what follows we assume the following brane set-up: the cycle $\tau_{s}$ is wrapped by a stack of magnetised D7-branes where appropriate gauge fluxes give rise to two different gauge groups that separately undergo gaugino condensation. This gives rise to a race-track superpotential. Due to the existence of a Euclidean D3-instanton wrapping the fibre $\tau_{f}$, this racetrack superpotential acquires further corrections. Following [24], the gauge kinetic functions of the two condensing gauge groups receive non-perturbative corrections, resulting 
in a poly-instanton corrected superpotential of the form:

$$
W=W_{0}+A e^{-a\left(T_{s}+C_{1} e^{-2 \pi T_{f}}\right)}-B e^{-b\left(T_{s}+C_{2} e^{-2 \pi T_{f}}\right)},
$$

Here, $A$ and $B$ are threshold effects, while $a=2 \pi / n_{a}$ and $b=2 \pi / n_{b}$ with $n_{a, b}$ natural numbers. $C_{1}$ and $C_{2}$ are constants associated with the effects of the D3-instanton wrapping $\tau_{f}$. On the other hand, the $\alpha^{\prime}$ corrected Kähler potential reads:

$$
K=K_{0}+K_{\alpha^{\prime}} \simeq-2 \ln \mathcal{V}-\frac{\xi}{g_{s}^{3 / 2} \mathcal{V}},
$$

where $\xi$ is a positive topological quantity.

The scalar potential computed from eqs. (3.1) and (3.2) can be written as:

$$
V=V_{\mathcal{O}\left(\mathcal{V}^{-3}\right)}\left(\tau_{s}, \mathcal{V}\right)+V_{\mathcal{O}\left(\mathcal{V}^{-3-p}\right)}\left(\tau_{f}, \tau_{s}, \mathcal{V}\right)
$$

where the first term scales with the volume as $\mathcal{V}^{-3}$, while the other as $\mathcal{V}^{-3-p}$, with $p$ a positive, order one parameter. If $\mathcal{V}$ is large, the second piece is much suppressed with respect to the first one: the previous equation reproduces the split between leading and subleading potentials we discussed in section 2 (see eq. (2.6)). The leading contribution to the scalar potential, $V_{\mathcal{O}\left(\mathcal{V}^{-3}\right)}$, is the standard LVS potential that stabilises the blow-up modulus $\tau_{s}$ and the volume mode $\mathcal{V}$ :

$$
\begin{aligned}
V_{\mathcal{O}\left(\mathcal{V}^{-3}\right)}= & \frac{8 \sqrt{\tau_{s}}\left(A^{2} a^{2} e^{-2 a \tau_{s}}+B^{2} b^{2} e^{-2 b \tau_{s}}-2 A B a b e^{-(a+b) \tau_{s}}\right)}{3 \mathcal{V}} \\
& +\frac{4 W_{0} \tau_{s}\left(A a e^{-a \tau_{s}}-B b e^{-b \tau_{s}}\right)}{\mathcal{V}^{2}}+\frac{3 \xi W_{0}^{2}}{4 g_{s}^{3 / 2} \mathcal{V}^{3}}
\end{aligned}
$$

The subleading $V_{\mathcal{O}\left(\mathcal{V}^{-3-p}\right)}$ term depends on all three directions of Kähler moduli space and generates a minimum for the fibre modulus $\tau_{f}$ :

$$
\begin{aligned}
V_{\mathcal{O}\left(\mathcal{V}^{-3-p}\right)=} & -e^{-2 \pi \tau_{f}}\left\{-\frac{16 C_{1} \sqrt{\tau_{s}} e^{-2 b \tau_{s}}}{3 \mathcal{V}}\left[Z^{2} a-Z B(a-b)\right]\right. \\
& +\frac{4 C_{1} W_{0} e^{-b \tau_{s}}}{\mathcal{V}^{2}}\left[2 \pi Z \tau_{f}+Z a \tau_{s}-B b(a-b) \tau_{s}\right] \\
& \left.+n\left[\frac{-16 B b^{2} \sqrt{\tau_{s}} e^{-2 b \tau_{s}} Z}{3 \mathcal{V}}+\frac{4 W_{0} B b e^{-b \tau_{s}}}{\mathcal{V}^{2}}\left(b \tau_{s}+2 \pi \tau_{f}\right)\right]\right\},
\end{aligned}
$$

where $Z \equiv B b-A a e^{-(a-b) \tau_{s}}$ and $n \equiv C_{2}-C_{1}$.

Given that the mass spectrum in the Kähler moduli sector exhibits the following hierarchy $m_{s}^{2} \sim \mathcal{V}^{-2} \gg m_{\mathcal{V}}^{2} \sim \mathcal{V}^{-3} \gg m_{f}^{2} \sim \mathcal{V}^{-3-p}$ with $p \sim \mathcal{O}(1)[9]$, we set $\tau_{s}$ and $\mathcal{V}$ to their minima determined (analytically) by minimising the potential (3.4):

$$
\left\langle\tau_{s}\right\rangle^{3 / 2}=\frac{3 \xi}{32 \alpha \gamma f_{1}\left(1-f_{1}\right) g_{s}^{3 / 2}}, \quad\langle\mathcal{V}\rangle=f_{2} e^{b\left\langle\tau_{s}\right\rangle} .
$$

Here we have defined:

$$
f_{1} \equiv \frac{(a-b) \tau_{s} B b+Z\left(1-a \tau_{s}\right)}{4(a-b) \tau_{s} B b+Z\left(1-4 a \tau_{s}\right)}, \quad \text { and } \quad f_{2} \equiv \frac{3 \alpha \gamma W_{0} \sqrt{\left\langle\tau_{s}\right\rangle}}{Z} f_{1} .
$$


This allows us to reduce the analysis of quintessence to the study of a single field potential for $\tau_{f}$, the subleading potential of eq. (3.5) where we substitute the VEVs of $\tau_{s}$ and $\mathcal{V}$. Following this procedure and performing the canonical normalisation of the fibre modulus (see appendix A, in particular the discussion around eq. (A.9)), the poly-instanton generated potential is written as:

$$
V_{\text {poly }}=\frac{F_{\text {poly }}}{\langle\mathcal{V}\rangle^{3+p}}\left[1-e^{-c\left(e^{\frac{2}{\sqrt{3}} \hat{\phi}}-1\right)}\left(1+c\left(e^{\frac{2 \hat{\phi}}{\sqrt{3}}}-1\right)\right)\right],
$$

where $\hat{\phi}$ is the field parameterising the shift of the canonically normalised fibre modulus from its minimum: $\hat{\phi} \equiv \phi-\langle\phi\rangle=\sqrt{3} / 2 \ln \left(1+\hat{\tau}_{f} /\left\langle\tau_{f}\right\rangle\right)$. The constant $c$ is given by $c=2 \pi\left\langle\tau_{f}\right\rangle=$ $p b\left\langle\tau_{s}\right\rangle+1 . F_{\text {poly }}$, on the other hand, is given in terms of the underlying parameters as:

$$
F_{\text {poly }}=\left(\frac{3 \alpha W_{0} \sqrt{\left\langle\tau_{s}\right\rangle} f_{1}}{Z}\right)^{p+1} 4 e^{-1} W_{0}\left(C_{1} Z-n B b\right) .
$$

Equation (3.8) provides a first, extremely flat contribution to the quintessence potential we will be working with. Note that, as explained in section 2, we included in eq. (3.8) an uplifting term, $V_{\text {up }}=F_{\text {poly }} /\langle\mathcal{V}\rangle^{3+p}$ so to set the minimum of the quintessence potential to zero.

As pointed out in [9], there will be further contributions to the fibre modulus potential, in particular UV physics will add new $\phi$ dependent terms to the total potential. These are loop contributions that we have to take into account, since they can influence the dynamics of the quintessence field. These contributions can be estimated via the Coleman-Weinberg potential and are due to subleading $g_{s}$ corrections to the Kähler potential. Supersymmetry reduces their size, and following the straightforward estimate performed in [9], we obtain:

$$
V_{\text {loop }} \simeq \frac{F_{\text {loop }}}{\langle\mathcal{V}\rangle^{4}} e^{\frac{2}{\sqrt{3}} \hat{\phi}}, \quad \text { where } \quad F_{\text {loop }}=\frac{c}{2 \pi}\left(g_{s} C_{\text {loop }} W_{0}\right)^{2} .
$$

The full potential for the fibre modulus that corresponds to our quintessence field is then given by:

$$
V_{\text {fibre }}=V_{\text {poly }}+V_{\text {loop }},
$$

which we plot in figure 1. Given that the gravitino mass in terms of the overall volume is $m_{3 / 2} \sim M_{P} / \mathcal{V}$, this potential for $p \sim \mathcal{O}(1)$ scales as $V_{\text {fibre }} \sim\left(M_{P} / \mathcal{V}\right)^{4} \sim m_{3 / 2}^{4}$, reproducing the behaviour described in section 2 .

For the study of the dynamics of the quintessence field it is convenient to divide the potential into three distinct regions:

1. The region I corresponds to the vicinity of the minimum where the potential is very steep;

2. The region II is the poly-instanton dominated part of the potential;

3. In region III the loop corrections are the dominant contribution to $V_{\text {fibre }}$.

Notice that, as outlined in section 2, we have obtained an extremely flat quintessential potential (above all in region II) in which supersymmetry in the extra-dimensions allows to tame 


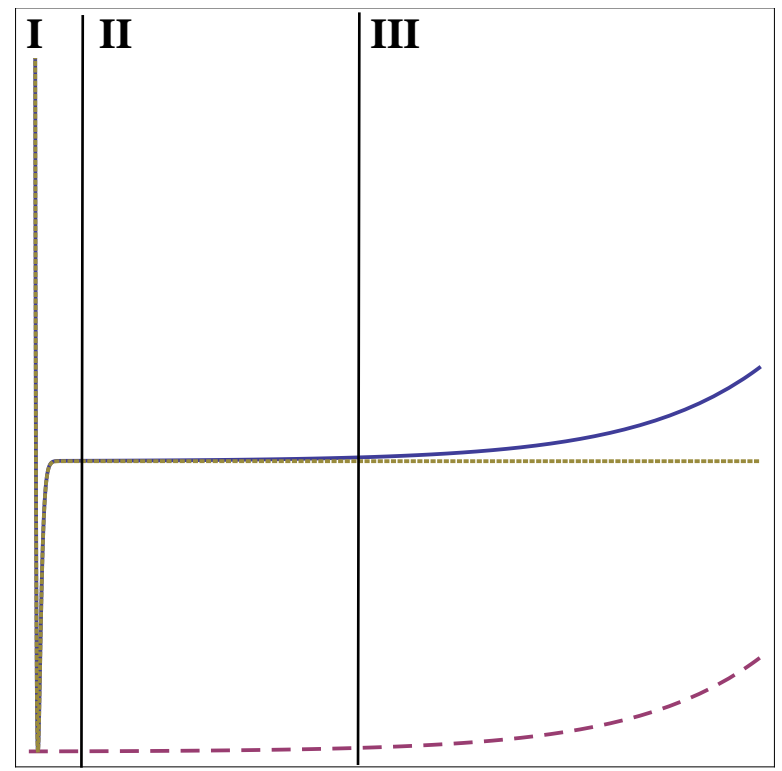

Figure 1. Quintessence potential for the fibre modulus. The dotted line represents the poly-instanton generated potential, the dashed line the loop potential from UV physics and the full line the total potential for the canonically normalised fibre modulus.

the dangerous corrections that would spoil its flatness. The aim of section 5 of this paper is to investigate whether the dynamics of the fibre modulus rolling down regions II and III gives a good description of the observed late time acceleration of Universe.

Let us conclude this section determining the relevant scales in our set-up. As we explained, observations constrain the dark-energy scale to be of the order $\rho_{D E} \sim 10^{-120} M_{P}^{4}$. In order to use the fibre modulus as a dynamical model of dark-energy, this maps into the following condition on the amplitude of the potential:

$$
\frac{F_{\text {poly }}}{\langle\mathcal{V}\rangle^{3+p}} \sim 10^{-121}
$$

This scale can be achieved by considering compactifications with very large volumes, as originally noted in [10], in particular for $p \sim \mathcal{O}(1)$ this yields:

$$
\langle\mathcal{V}\rangle \sim 10^{30}
$$

in string units. We stress that, in our scenario, the VEV of the volume depends exponentially on the microscopic parameters of the model (see eq. (3.6)), and such large values are easy to obtain. So, once the minimum of the quintessence potential is set to zero, the tiny height of the potential, eq. (3.12), is obtained straightforwardly! Notice that values of the volume of the order $\mathcal{V} \sim 10^{30}$ correspond to a string scale of the order the TeV-scale, $M_{s} \sim M_{P} / \sqrt{\mathcal{V}} \sim \mathcal{O}(\mathrm{TeV})$, and a gravitino mass of the order the cosmological constant scale, $m_{3 / 2} \sim M_{P} / \mathcal{V} \sim \mathcal{O}(\mathrm{meV})$, according to the discussion of section 2. Such a low-value of the fundamental gravity scale poses a severe problem for obtaining the original period of inflation since both the amount of density perturbations and the resulting reheating temperature would be too low. For example, the inflationary model developed in [9] requires values of the volume of the order $\mathcal{V} \sim 10^{3}$ corresponding to a GUT-scale $M_{s}$. The general idea that we have in 
mind to overcome this problem is to use the volume mode as the inflaton following the work of [25]. Thus the volume can take smaller values of the order $\mathcal{V} \sim 10^{3}$ in the early Universe, and then roll to values of the order $\mathcal{V} \sim 10^{30}$ after the end of inflation, where the only field left over to roll down its minimum is the fibre modulus $\tau_{f}$ which drives quintessence.

A very large volume, apart from dynamically describing the smallness of the observed size of dark energy, has also the virtue to suppress fifth-force couplings between the quintessence field and SM matter, and to enlarge the very flat region II in the potential $V_{\text {fibre }}$. In the next sections, we will discuss these features in detail.

\section{Quintessence coupling to matter}

The first step in the computation of the quintessence couplings to ordinary matter is the moduli canonical normalisation, which we perform in detail in appendix A. The expression of the original moduli $\tau_{f}, \mathcal{V}$, and $\tau_{s}$ in terms of the corresponding canonically normalised fields $\phi, \chi_{\mathcal{V}}$ and $\chi_{s}$ can be obtained explicitly at leading order in an inverse volume expansion. Considering all the moduli in the vicinity of their minimum, the canonical normalisation of the Standard Model modulus represented by $\tau_{s}$, takes the form (see eq. (A.10)):

$$
\frac{\delta \tau_{s}}{\left\langle\tau_{s}\right\rangle} \sim \mathcal{O}\left(\mathcal{V}^{-p}\right) \delta \phi+\mathcal{O}(1) \delta \chi_{\mathcal{V}}+\mathcal{O}(\sqrt{\mathcal{V}}) \delta \chi_{s},
$$

where $\phi$ is the quintessence field, while $\chi_{\mathcal{V}}$ and $\chi_{s}$ are two heavy fields corresponding respectively to the volume mode and the small rigid divisor $\tau_{s}$. The exact coefficients of (4.1) will be computed in appendix A at leading order in an inverse volume expansion. What is crucial, in the previous expression, is that the canonically normalised quintessence field $\phi$ provides only a suppressed contribution to $\delta \tau_{s}$, essentially determined by the ratio of the leading and subleading scalar potentials of eqs (2.7) and (2.8) (recall also the general discussion of section 2.2). Since $\tau_{s}$ controls the properties of SM physics, this implies that the coupling of the quintessence field to Standard Model particles is very suppressed.

Before passing to prove this fact explicitly, let us discuss a technical issue: during the quintessence dynamics the two heavy fields $\chi_{\mathcal{V}}$ and $\chi_{s}$ sit at their minima, but the rolling scalar $\phi$ can be far away from its minimum. Hence the fibre modulus has to be canonically normalised not just close to its minimum but for an arbitrary point in moduli space. This is a complicated computation whose result has already been qualitatively discussed in [26] where the authors used the poly-instanton effects for deriving a model of modulated reheating. The final upshot is that in regions far away from the minimum for large values of $\tau_{f}$, the poly-instantons get more suppressed, and so the mixing with the other moduli also gets more suppressed. Thus the coupling of the fibre modulus to ordinary matter gets weaker in regions far from the minimum. In fact, if $\tau_{f}$ is far from its minimum then:

$$
e^{-2 \pi \tau_{f}}=e^{-2 \pi\left\langle\tau_{f}\right\rangle} e^{-2 \pi \hat{\tau}_{f}} \sim \mathcal{V}^{-p} e^{-2 \pi \hat{\tau}_{f}},
$$

and so the mixing term in (4.1) would be even more suppressed than $\mathcal{V}^{-p}$.

\subsection{Coupling to gauge bosons}

We start by estimating the couplings to gauge bosons following [10]. Recalling that $\tau_{s}$ is the Standard Model cycle, the gauge kinetic terms are given by:

$$
\mathcal{L}_{\text {gauge }}=-\frac{\tau_{s}}{M_{P}} F_{\mu \nu} F^{\mu \nu} .
$$


After expanding $\tau_{s}$ around the minimum $\left(\tau_{s}=\left\langle\tau_{s}\right\rangle+\delta \tau_{s}\right)$ and performing the canonical normalisation of the field strength tensor, we find that the interaction term is:

$$
\mathcal{L} \supset \frac{\delta \tau_{s}}{4 M_{P}\left\langle\tau_{s}\right\rangle} G_{\mu \nu} G^{\mu \nu} .
$$

From eq. (4.1) it follows that:

$$
\frac{\delta \tau_{s}}{\left\langle\tau_{s}\right\rangle} \sim \mathcal{O}\left(\mathcal{V}^{-p}\right) \delta \phi
$$

and so we find:

$$
\mathcal{L} \supset \mathcal{O}\left(\frac{1}{M_{P} \mathcal{V}^{p}}\right) \delta \phi G_{\mu \nu} G^{\mu \nu} .
$$

We conclude that the coupling between the fibre modulus and the SM gauge bosons is weakerthan-Planckian for $p \sim \mathcal{O}(1)$, in accordance with [10], and therefore poses no issues with the constraints from fifth-force experiments.

\subsection{Coupling to fermions}

Let us now turn our attention to the coupling between the quintessence field and the Standard Model fermions, which we denote as $C_{i}$. The relevant part of the SUGRA Lagrangian for the computation of this coupling is:

$$
\mathcal{L} \supset \tilde{K}_{i j} \overline{C_{i}} i \gamma^{\mu} \partial_{\mu} C_{j}+\tilde{K}_{H \bar{H}} \partial^{\mu} H \partial_{\mu} \bar{H}+e^{K / 2} \lambda_{i j} C_{i} C_{j} H,
$$

where $\tilde{K}_{i j}$ and $\tilde{K}_{H \bar{H}}$ are generic functions of the moduli fields. Very little can be said about this theory without specifying $\tilde{K}_{i j}$ and $\tilde{K}_{H \bar{H}}$, and so we follow the work of [27], where the leading modular dependence of these two functions has been estimated through simple scaling arguments. We simplify the set-up further by considering diagonal Kähler metrics and Yukawa couplings:

$$
\mathcal{L} \supset \tilde{K}_{i i} \bar{C}_{i} i \gamma^{\mu} \partial_{\mu} C_{i}+\tilde{K}_{H \bar{H}} \partial^{\mu} H \partial_{\mu} \bar{H}+e^{K / 2} \lambda \overline{C_{i}} C_{i} H .
$$

Following [27] we take:

$$
\tilde{K}_{i i} \sim \tilde{K}_{H \bar{H}} \sim \frac{\tau_{s}^{1 / 3}}{\mathcal{V}^{2 / 3}} .
$$

Expanding $\tau_{s}$ and $\mathcal{V}$ around their VEVs as $\tau_{s}=\left\langle\tau_{s}\right\rangle+\delta \tau_{s}$ and $\mathcal{V}=\langle\mathcal{V}\rangle+\delta \mathcal{V}$ we can write the Kähler metrics as:

$$
\tilde{K}_{i i} \sim \tilde{K}_{H \bar{H}} \sim \tilde{K}_{0}\left(1+\frac{\delta \tau_{s}}{3\left\langle\tau_{s}\right\rangle}-\frac{2 \delta \mathcal{V}}{3\langle\mathcal{V}\rangle}\right) \quad \text { where } \quad \tilde{K}_{0} \equiv \frac{\left\langle\tau_{s}\right\rangle^{1 / 3}}{\langle\mathcal{V}\rangle^{2 / 3}}
$$

This allows us to define the canonically normalised fields $c_{i}=\sqrt{\tilde{K}_{0}} C_{i}$ and $h=\sqrt{\tilde{K}_{0}} H$ and rewrite the Lagrangian as:

$$
\mathcal{L} \supset\left(1+\frac{\delta \tau_{s}}{3\left\langle\tau_{s}\right\rangle}-\frac{2 \delta \mathcal{V}}{3\langle\mathcal{V}\rangle}\right) \overline{c_{i}} i \gamma^{\mu} \partial_{\mu} c_{i}+\partial^{\mu} h \partial_{\mu} \bar{h}+\frac{\lambda}{\mathcal{V} \tilde{K}_{0}^{3 / 2}} \overline{c_{i}} c_{i} h
$$

Setting $h=\langle h\rangle$, expanding $1 / \mathcal{V} \sim 1 /\langle\mathcal{V}\rangle(1-\delta \mathcal{V} /\langle\mathcal{V}\rangle)$ and defining the fermionic mass as $m_{c} \equiv \frac{\lambda\langle h\rangle}{\left\langle\tau_{s}\right\rangle^{1 / 2} \tilde{K}_{0}^{3 / 2}}$ this becomes:

$$
\mathcal{L} \supset \overline{c_{i}}\left(i \gamma^{\mu} \partial_{\mu}+m_{c}\right) c_{i}+\left(\frac{\delta \tau_{s}}{3\left\langle\tau_{s}\right\rangle}-\frac{2 \delta \mathcal{V}}{3\langle\mathcal{V}\rangle}\right) \overline{c_{i}}\left(i \gamma^{\mu} \partial_{\mu}+m_{c}\right) c_{i}-m_{c} \overline{c_{i}} c_{i}\left(\frac{\delta \tau_{s}}{3\left\langle\tau_{s}\right\rangle}+\frac{\delta \mathcal{V}}{3\langle\mathcal{V}\rangle}\right)
$$


Note that the first term is the standard Lagrangian for a free fermion of mass $m_{c}$, the second term vanishes once we impose the equations of motion and the third term encodes the interaction between moduli and Standard Model fermions. Expressing the moduli in terms of the canonically normalised fields near the minimum, we find that the dominant term in the interaction with the quintessence field comes from the expression (4.1) for $\delta \tau_{s}$ term, since to leading order $\delta \mathcal{V}$ does not depend on the canonically normalised fibre modulus. This implies that the leading contribution to the dimensionless coupling between ordinary fermions and the quintessence field is:

$$
\lambda_{\phi \bar{c} c} \sim \mathcal{O}\left(\frac{m_{c}}{M_{P} \mathcal{V}^{p}}\right)
$$

which is weaker-than-Planckian for $p \sim \mathcal{O}(1)$ like the coupling to Standard Model gauge bosons.

The fact that the couplings to gauge bosons and fermions are suppressed by a scale higher than the Planck mass means that the current model is able to comfortably accommodate the constraints from fifth-force experiments, see e.g. [3], and provide a controlled string theoretical description of quintessence.

\section{Dynamics of the quintessence-dark matter system}

In this section we study the dynamics of the quintessence-dark matter system by following the autonomous phase plane analysis originally developed in [28]. We start by analysing a model in which we neglect any direct coupling between the two dominant components of the energy density (this since their direct coupling is weaker-than-Planckian). Then we look at the case where there is a direct, Planck strength coupling between dark matter and dark energy, while at the same time dark energy couples extremely weakly to ordinary matter. In this way, we will outline a proposal of how to realise a coupled quintessence scenario within string theory.

We consider a flat FRW Universe filled with quintessence as described by the fibre modulus and a pressureless dark matter component. The time evolution of the system is determined by the Raychaudhuri equation, the continuity equation for the dark matter component, the Klein-Gordon equation for a homogeneous scalar field and the Friedmann constraint:

$$
\begin{array}{r}
\dot{H}=-\frac{1}{2 M_{P}^{2}}\left(\rho_{D M}+\dot{\phi}^{2}\right), \\
\dot{\rho}_{D M}=-3 H \rho_{D M}+Q, \\
\ddot{\phi}+3 H \dot{\phi}+\frac{d V}{d \phi}=\frac{Q}{\dot{\phi}} \\
\Omega_{D M}+\Omega_{D E}=1,
\end{array}
$$

where $\Omega \equiv \frac{\rho}{3 H^{2} M_{P}^{2}}$ and $Q$ parameterises the direct interaction between the dark matter and the quintessence components. Using $N=\ln a$ as the time variable and defining:

$$
x \equiv \frac{\phi^{\prime}}{M_{P} \sqrt{6}}, \quad y^{2} \equiv \frac{V}{3 M_{P}^{2} H^{2}},
$$


the equations of motion can be cast in the following form:

$$
\begin{array}{r}
x^{\prime}(N)=-3 x-\frac{\partial V / \partial \phi}{V} \sqrt{\frac{3}{2}} y^{2}+\frac{3}{2} x\left(2 x^{2}+\left(1-x^{2}-y^{2}\right)\right)+\frac{Q}{6 H^{3} M_{P}^{2} x}, \\
y^{\prime}(N)=\frac{\partial V / \partial \phi}{V} \sqrt{\frac{3}{2}} x y+\frac{3}{2} y\left(2 x^{2}+\left(1-x^{2}-y^{2}\right)\right), \\
H^{\prime}(N)=-\frac{3 H}{2}\left(2 x^{2}+\left(1-x^{2}-y^{2}\right)\right), \\
\phi^{\prime}(N)=\sqrt{6} x .
\end{array}
$$

In what follows we will both search analytically for instantaneous fixed points of the system and study the phase space trajectories numerically.

\subsection{Decoupled quintessence and dark matter}

We first analyse the case where a direct coupling between dark matter and dark energy is negligible, which corresponds to setting $Q=0$. The system possesses two non trivial instantaneous fixed points, $x^{\prime}(N)=y^{\prime}(N)=0$, whose properties are listed in table 1 . These correspond to motion in the two distinct regions of the potential: point $\mathcal{A}$ corresponds to motion in the poly-instanton dominated part of the potential (labelled II in figure 1) whereas point $\mathcal{B}$ describes motion in the region where the loop potential dominates (labelled region III in figure 1). Note that the flatness of the poly-instanton potential implies that $\delta \equiv V_{\text {poly }}^{\prime} / V_{\text {poly }} \sim 0$, and so the behavior of the fibre modulus approaches that of a pure cosmological constant.

\begin{tabular}{c|c|c|c|c} 
Point & $\left(x_{c}, y_{c}\right)$ & Stability & $\Omega_{\phi}$ & $\omega_{\phi}$ \\
\hline $\mathcal{A}$ & $\left(\delta, \sqrt{1-\delta^{2}}\right)$ & stable node & 1 & -1 \\
$\mathcal{B}$ & $(2 / \sqrt{18}, \sqrt{14 / 18})$ & stable node & 1 & $-5 / 9$ \\
\hline
\end{tabular}

Table 1. Fixed points for the quintessence dark-matter system.

From the definitions of eq. (5.5), one sees that $x(N)(y(N))$ is the ratio of the scalar field's kinetic (potential) energy to the total energy. We illustrate the behaviour of the system in figure 2 for different sets of initial conditions. The region above the parabola corresponds to accelerating solutions and the dots represent the two instantaneous fixed points described in table 1.

We see that if the fibre modulus starts its evolution in the part of the potential that is dominated by the loop effects, the system will evolve towards the fixed point $\mathcal{B}$. This fixed point corresponds to an accelerated expansion solution, where the energy density is dominated by the scalar field, as preferred by current cosmological data. However the equation of state parameter, which is generically given by:

$$
\omega_{\phi}=\frac{x-y}{x+y}
$$

is different from 1 for the fixed point $\mathcal{B}$. Thus, given the current observational constraints, this fixed point is not a viable candidate for dark energy. As the fibre modulus rolls down the loop generated potential it eventually enters a region in field space where the loop potential is suppressed relative to the poly-instanton generated potential. In this region the relevant fixed 

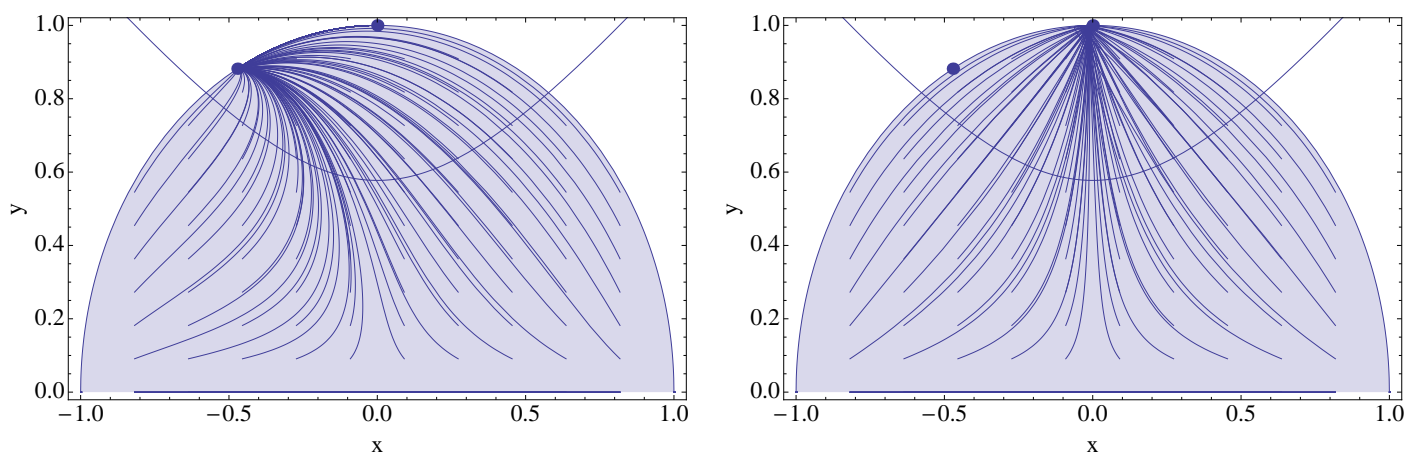

Figure 2. Phase plane evolution of the quintessence dark-matter system. Left: the fibre modulus starts in the loop dominated region of the potential; right: the fibre modulus starts in the polyinstanton dominated region of the potential.

point to which the system will converge is $\mathcal{A}$, which corresponds to slow roll dynamics in the poly-instanton dominated part of the potential. The fixed point $\mathcal{A}$ describes a flat Universe filled with a negative pressure fluid with $\omega=-1$. Even though such trajectories display the same asymptotic behaviour in the future as the observed Universe, a numerical analysis of the system suggests they never go through a phase in which the conditions $\Omega_{D E} \sim 0.7$ and $\omega_{\phi} \sim-1$ are simultaneously verified. It then follows that trajectories that start with the quintessence field deep in the loop dominated region of potential do not provide a viable description of the late time Universe. On the other hand, if the initial conditions are such that the fibre modulus starts its evolution in a region of the potential where the loop effects are negligible, it will evolve towards the fixed point $\mathcal{A}$ without going through $\mathcal{B}$ first. As can be seen in figure 2, these trajectories can go through the region of phase space where the energy density and the equation of state parameter of the quintessence field are in agreement with observational data. We then conclude that there are phase space trajectories for which the system evolves to a state where it provides a viable description of an accelerated expanding Universe, namely the ones corresponding to initial field values in the poly-instanton plateau. It is worth noting that from the discussion in section 3 the width of the poly-instanton plateau is determined by the ratio $V_{\text {poly }} / V_{\text {loop }} \sim \mathcal{V}^{1-p}$ which can easily be $\gg 1$ given the large volumes required for quintessence phenomenology, $\mathcal{V} \sim 10^{30}$. There is therefore an appreciable range of initial conditions for the fibre modulus that yield a viable quintessential behaviour.

When looking at the late time composition of the Universe one is confronted with the coincidence problem: why is the dark energy density comparable to the dark matter density today? Dynamical models of dark energy often address this issue by allowing for scaling solutions: fixed points where the ratio of dark energy to dark matter density is constant. The existence of these scaling solutions is determined by the steepness of the dark-energy potential $\left(V^{\prime} / V\right)$ and by the equation of state for the matter component. In a Universe with pressureless cold dark matter and the fibre modulus playing the rôle of quintessence, there are no such fixed points as both regions of the quintessence potential are too shallow to support them. Therefore we are led to the conclusion that even though our model provides a realistic description of the current state of the Universe, it does not allow for a dynamical explanation of the coincidence problem: we just happen to be around at a time when $\Omega_{D E}$ and $\Omega_{D M}$ are of the same order and the Universe will eventually evolve to a state where it is 
filled with quintessence.

In these considerations about the distant future evolution of the system we have so far ignored the presence of the minimum of the potential, region I in fig. 1, to the left of the poly-instanton plateau. Its presence implies that in the very distant future the fibre modulus will eventually fall into its minimum, after which will follow a phase of oscillatory dynamics, in a process similar to post-inflationary reheating, albeit at a much lower energy scales. Since, ultimately, acceleration ends with the scalar sitting at its Minkowski minimum, we avoid the problems raised in $[29,30]$ with respect of embedding asymptotic de Sitter space in string theory.

\subsection{Coupled quintessence and dark matter}

We now analyse how a coupled quintessence-dark matter system affects the dynamics of the Universe [31]. Possible Planck suppressed couplings between dark energy and dark matter are allowed in our set-up if dark matter is constituted by bulk KK modes or moduli fields.

Let us start discussing possible dark matter candidates: phenomenological constraints on dark matter from collider physics, astrophysics and cosmology in this class of scenarios have been discussed in [10]. Here we briefly summarise the main conclusions. The requirement of avoiding BBN constraints, associated with the fact that the SM brane cools too quickly due to evaporation of energy into the bulk, sets a severe bound on the reheating temperature: $T_{\mathrm{RH}} \lesssim 100 \mathrm{MeV}$. If this constraint is satisfied, there are no issues with $\mathrm{BBN}$, but the relic KK modes, if stable, can still overclose the Universe. In order to avoid this, the previous bound becomes more severe, bringing the reheating temperature at most around a few $\mathrm{MeV}$, just above the BBN temperature. On the other hand, if the KK modes are not stable and decay into photons, then one can have problems with possible distortions of the diffuse $\mathrm{MeV}$ $\gamma$-ray background.

Notice that these bounds on the reheating temperature imply that we cannot have a thermal bath up to the weak scale, and so the standard thermal WIMP picture for dark matter does not work. In addition, we do not have neutralini in the 4D low-energy spectrum since supersymmetry is badly broken on the SM brane. In our case we have two kinds of KK modes: 6D KK modes and 10D KK modes. The 10D KK modes decay whereas the 6D KK modes are so long lived that can be considered as almost stable with respect to the age of the Universe. Hence given that the $6 \mathrm{D}$ KK modes are almost stable, we need to have a reheating temperature just above the $\mathrm{MeV}$ scale. The advantage is that the $10 \mathrm{D}$ KK modes decay predominantly to $6 \mathrm{D}$ KK modes, and so their decay to photons is suppressed. Thus we have no problems associated with $\gamma$-ray background distortion.

Higher 6D KK states can be produced from the thermal bath but they quickly decay to lower 6D KK states, which are then long-lived. There are also two light moduli which are stable with respect to the age of the Universe. One is the volume mode which we can suppose to be the inflaton, so that inflation takes place when the gravity scale is high, whereas at the end of inflation, the string scale is around the TeV. This implies that the volume mode is very light with a mass of the order meV. Hence this modulus can be produced in the thermal bath (or by simple scalar oscillation) and then suffers from the cosmological moduli problem (CMP). The other light modulus is the fibre divisor which drives quintessence.

Therefore, we conclude that in our scenario dark matter can be realised in terms of a mix of bulk $6 \mathrm{D}$ KK modes and the volume modulus, once the CMP associated with the latter is solved (for example via a second low-energy inflationary period as in thermal inflation). The quintessence field would then indeed couple to dark matter, for example 6D KK modes, 
with Planck suppressed trilinear metric couplings of the form:

$$
\frac{\psi}{M_{P}} \partial \phi \partial \phi,
$$

where $\phi$ is the quintessence field and $\psi$ the $6 \mathrm{D}$ KK mode.

After this qualitative discussion to motivate the coupling of dark energy to dark matter in our set-up, we can write the dark matter energy density as a function of the fibre modulus as:

$$
\rho_{D M}=\rho_{0} f(\phi),
$$

where $\rho_{0}$ obeys the normal conservation equation:

$$
\dot{\rho}_{0}=-3 H \rho_{0},
$$

and $f(\phi)$ parameterises the interaction between dark matter and the quintessence field. The conservation equation for dark matter is then given by:

$$
\dot{\rho}_{D M}=-3 H \rho_{D M}+\frac{\partial f}{\partial \phi} \frac{\dot{\phi}}{f(\phi)} \rho_{D M} .
$$

By comparing eqs. (5.2) and (5.14) we define $Q \equiv-\frac{\partial f}{\partial \phi} \frac{\dot{\phi}}{f(\phi)} \rho_{D M}$. In order to look for fixed points analytically, one must be able to write $\frac{\partial f}{f(\phi) \partial \phi}$ in terms of $x(N), y(N)$ and other dimensionless parameters. One simple case might be:

$$
f(\phi)=e^{\alpha \phi / M_{P}},
$$

since this form for the coupling function implies that:

$$
\frac{\partial f}{\partial \phi} \frac{\dot{\phi}}{f(\phi)} \rho_{D M}=\frac{\alpha}{M_{P}} \dot{\phi} \rho_{D M},
$$

allowing for an analytical study of the phase plane dynamics of the system. This is exactly what one expects to find if the dark matter mass is a function of the fibre modulus $\tau_{f}$,

$$
m_{D M} \sim \tau_{f}^{m} \sim e^{2 m \phi / \sqrt{3}}
$$

where in the last step we have written $\tau_{f}$ in terms of the canonically normalised field $\phi$.

It then follows that $Q=-\alpha \sqrt{6} x H \rho_{D M}$ and eq. (5.6) becomes:

$$
x^{\prime}(N)=-3 x-\frac{\partial V / \partial \phi}{V} \sqrt{\frac{3}{2}} y^{2}+\frac{3}{2} x\left(2 x^{2}+\left(1-x^{2}-y^{2}\right)\right)-\alpha \sqrt{3 / 2}\left(1-x^{2}-y^{2}\right) .
$$

The phase space for models with this form of the direct coupling $\mathrm{Q}$ has been studied in detail in [32], where a phenomenologically interesting fixed point located at $\left(x_{c}, y_{c}\right)=$ $\left(\frac{\sqrt{6}}{2 b}, \frac{\sqrt{9+6 \alpha b}}{\sqrt{6} b}\right)$ was found. Note that we have defined $\lambda \equiv-M_{P} \frac{\partial V}{V \partial \phi}$ and $b \equiv \lambda+\alpha$. Following [32] we find that this fixed point exists if:

$$
b^{2} \geq \frac{3}{2} \quad \text { and } \quad-\frac{3}{2} \leq \alpha b \leq b^{2}-3
$$


and is stable if:

$$
b^{2} \geq \frac{3}{2} \quad \text { and } \quad\left(-\frac{3}{2}<\alpha b<-B_{\operatorname{sgn}(b)} \quad \text { or } \quad-B_{-\operatorname{sgn}(b)}<\alpha b<b^{2}-3\right),
$$

where:

$$
B_{ \pm}=\frac{2 b\left[ \pm\left(b^{2}-3 / 2\right)^{3 / 2}-b\left(b^{2}-39 / 8\right)\right]}{4 b^{2}+3 / 4} .
$$

As in the decoupled case, we proceed by considering two different regimes, depending on which term in the potential dominates. For each case we shall then investigate whether the above conditions are met. In the regime in which the poly-instanton effects dominate over the loop potential (region II of figure 1), $\lambda \sim 0$ to a very good accuracy. One sees that it is impossible to satisfy the existence conditions given by eq. (5.19). We then conclude that the poly-instanton potential is unable to support an interacting tracking solution. However if one looks at the regime where the loop generated potential dominates (region III of figure 1 ), one has $\lambda=-2 / \sqrt{3}$ and one sees that there are values of $\alpha$ for which a stable accelerating tracking solution exists. This region however is not very wide due to the requirement of stability. In fact we find that it is roughly $-\frac{5}{2 \sqrt{3}}-0.55<\alpha<-\frac{5}{2 \sqrt{3}}$. We must note that this is not so much a consequence of the shape of the potential as of the explicit form of $Q$ we are assuming. Furthermore, we see that in this narrow stability region $0.93<\Omega_{D E}<1$, where the lower bound corresponds to $\alpha=-\frac{5}{2 \sqrt{3}}-0.55$ and the upper bound to $\alpha=-\frac{5}{2 \sqrt{3}}$. It is worth noting that for $\alpha<0$ the dark matter mass goes like $m_{D M} \propto 1 / \tau_{f}^{m}$.

The behavior described above is illustrated in fig. 3, where it is clear that the presence of the interaction dramatically changes the trajectories in phase space. One also sees that the coupled system (magenta) converges to a fixed point which is different from the one of the decoupled case (blue lines).
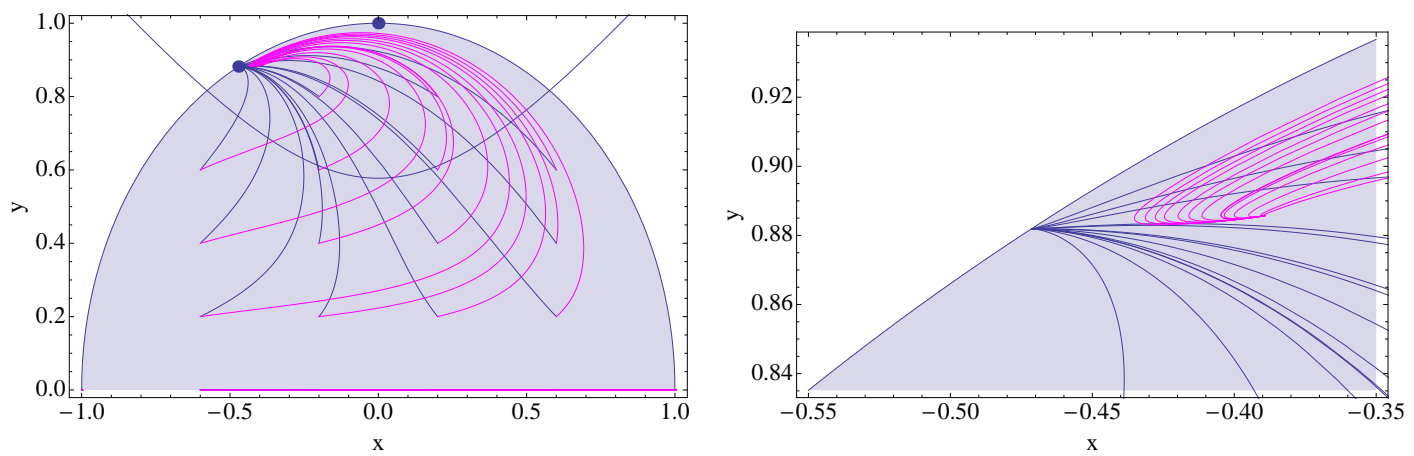

Figure 3. Phase plane evolution of the quintessence dark-matter system with interaction and $F_{\text {poly }} \gg$ $F_{\text {loop }}$

Once one considers the full potential, the fixed point described above is transient since as the field rolls down the loop generated potential it eventually enters the poly-instanton dominated region. So the phase-space trajectories start by converging to the fixed point $\mathcal{B}$ before evolving to the cosmological constant fixed point $\mathcal{A}$.

As in the non interacting case, the fibre modulus is able to provide suitable description of dark energy even though it does not address the coincidence problem. The introduction of the non minimal interaction term extends the existence of viable trajectories for cases when the field starts its evolution deep in the loop dominated part of the potential. 

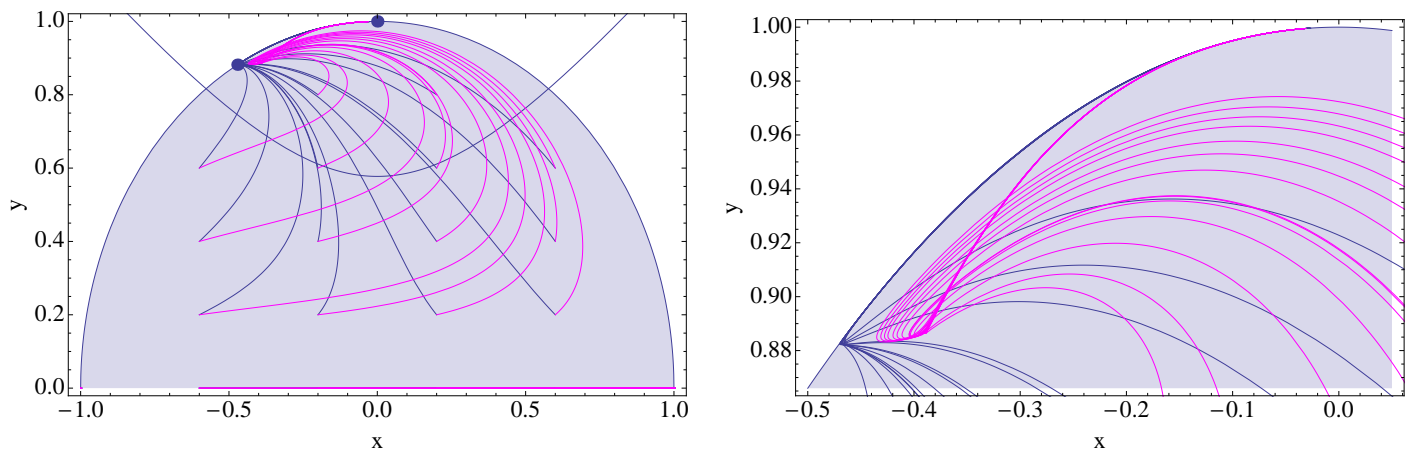

Figure 4. Phase plane evolution of the quintessence dark-matter system with interaction and $F_{\text {poly }} \sim$ $10^{3} F_{\text {loop }}$

\section{Conclusions}

In this paper, we presented a new quintessence model embedded in string theory where the small mass of the rolling scalar field is technically natural and its coupling to Standard Model degrees of freedom is naturally weaker-than-Planckian, thus avoiding fifth-force constraints.

We focused on a type IIB LARGE Volume Scenario with a very anisotropic shape of the extra dimensions since two of them are stabilised at values exponentially larger than the other four [10]. This leads to a string derivation of SLED scenarios [11] which have already turned out to be very promising for obtaining models of quintessence [12]. In these scenarios, if TeV-scale gravity provides the solution to the hierarchy problem, ${ }^{4}$ the gravitino mass $m_{3 / 2}$ turns out to be of the observed order of magnitude of the cosmological constant: $m_{3 / 2} \sim \Lambda$. Therefore, the current value of dark energy $\Lambda$ can be naturally reproduced if the quintessence field develops a potential at order $V \sim m_{3 / 2}^{4}$.

This mechanism, which ties the solution of the hierarchy problem to the one of the cosmological constant problem, relies on the existence of a compensation between the SM brane tension and its back-reaction. A possibility to achieve this could be to directly apply to our set-up the ideas developed in 6D SLED constructions. This, in turn, depends on the details of the $6 \mathrm{D}$ effective supergravity theory for our anisotropic compactifications; on the other hand, this intermediate theory has not yet been explicitly identified in [10]. We leave a proper understanding of these interesting open issues for study in the near future.

In this paper, we showed that the fibre divisor $\tau_{f}$ indeed develops a potential at order $V \sim m_{3 / 2}^{4}$ via tiny poly-instanton corrections to the superpotential and closed string loop contributions to the Kähler potential. Furthermore, this field is radiatively stable since the bulk is nearly supersymmetric.

We also showed that our quintessence model can evade the severe bounds coming from fifth-force experiments even if we are dealing with a scalar. The reason is the fact that the rigid four-cycle supporting the Standard Model does not intersect the fibre divisor, and so the quintessence scalar couples to ordinary matter only via its mixing with the volume mode which, in turn, induces its mixing with the SM cycle. However due to the fact that the fibre modulus is a flat direction at leading order which is lifted only at subleading order, the

\footnotetext{
${ }^{4}$ Notice that all the scales of our model are set by the value of the overall volume $\mathcal{V}$ which has an exponential dependence on the underlying parameters, and so it can be easily set to arbitrarily large values without tunings.
} 
mixing between $\tau_{f}$ and $\mathcal{V}$ is suppressed, resulting in a weaker-than-Planckian coupling of the quintessence field to SM particles.

On the other hand, if dark matter is realised in terms of quasi-stable Kaluza-Klein states, direct couplings between dark energy and dark matter are allowed, leading to a scenario of coupled quintessence within string theory. We studied in detail the dynamics of the quintessence scalar in our set-ups, investigating the nature of fixed points and the late time evolution of dark energy, showing that the main features of our scenario make it compatible with observations.

To conclude, our model explicitly shows that concrete string theory constructions are able to lead to scalar theories of quintessence that, besides generating naturally flat scalar potentials, also provide couplings to Standard Model matter that are weak enough to satisfy present constraints on fifth forces.

\section{Acknowledgments}

We would like to thank Cliff Burgess, Fernando Quevedo and Joseph Conlon for useful discussions. GT is supported by an STFC Advanced Fellowship ST/H005498/1. FGP is supported by Fundação para a Ciência e Tecnologia (Portugal) through the grant SFRH/BD/35756/2007 and by the Theoretical Physics Department of the University of Oxford.

\section{A Canonical normalisation}

In this section we compute the canonical normalisation for the fields involved, using the well-known techniques developed in $[27,33-35]$. The Lagrangian for the quadratic field fluctuations around their minima, $\tau_{i}=\left\langle\tau_{i}\right\rangle+\delta \tau_{i}$, takes the form:

$$
\mathcal{L}=K_{i j} \partial_{\mu} \delta \tau_{i} \partial^{\mu} \delta \tau_{i}-\langle V\rangle-\frac{1}{2} V_{i j} \delta \tau_{i} \delta \tau_{j},
$$

where $K_{i j}$ is the Kähler matrix, and $V_{i j}$ the matrix of second derivatives of the potential. The leading terms in the Kähler matrix $K_{i j}$ look like:

$$
K_{i j} \simeq\left(\begin{array}{ccc}
\frac{3}{8 \tau_{f}^{2}} & -\frac{1}{4 \mathcal{V} \tau_{f}} & -\frac{3 \sqrt{\tau_{s}}}{8 \mathcal{V} \tau_{f}} \\
-\frac{1}{4 \mathcal{V} \tau_{f}} & \frac{1}{2 \mathcal{V}^{2}} & 0 \\
-\frac{3 \sqrt{\tau_{s}}}{8 \mathcal{V} \tau_{f}} & 0 & \frac{3}{8 \mathcal{V} \sqrt{\tau_{s}}}
\end{array}\right),
$$

where we used $\left(\tau_{f}, \mathcal{V}, \tau_{s}\right)$ as coordinates of the Kähler moduli space. We write the original moduli in terms of the canonically normalised fields around their minima as:

$$
\left(\begin{array}{l}
\delta \tau_{f} \\
\delta \mathcal{V} \\
\delta \tau_{s}
\end{array}\right)=\mathcal{C}\left(\begin{array}{c}
\delta \phi \\
\delta \chi_{\mathcal{V}} \\
\delta \chi_{s}
\end{array}\right)
$$

where $\mathcal{C}$ is the matrix that diagonalises both the kinetic and the mass terms. It can be written as:

$$
\mathcal{C}=\left(\begin{array}{lll}
\vec{v}_{(1)} & \vec{v}_{(2)} & \vec{v}_{(3)}
\end{array}\right)
$$


where $\vec{v}_{(i)}$ are the eigenvectors of the mass matrix $M_{i k}=\frac{1}{2} K_{i j}^{-1} V_{j k}$, normalised such that $\mathcal{C}_{i k}^{T} K_{k m} \mathcal{C}_{m j}=\delta_{i j}$. The mass matrix reads explicitly:

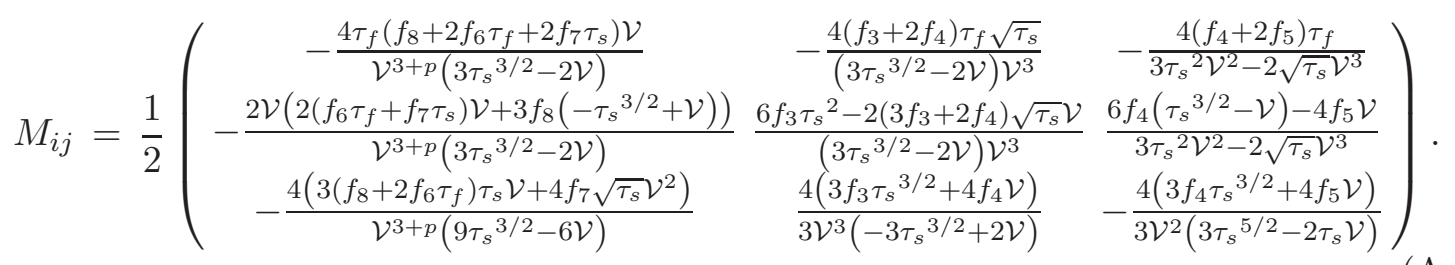

The quantities $f_{i}$ are independent from the volume at leading order in an inverse volume expansion. Their expressions are quite long, and we relegate them in appendix B. In writing the previous mass matrix, we added to the leading part of the potential, eq. (3.4), the subleading contribution due to polyinstanton corrections, eq. (3.5).

Notice that, as expected, the first column of the mass matrix is suppressed by a factor $1 / \mathcal{V}^{3+p}$, since it is due to the subleading poly-instanton potential. In other words, the first column of the matrix (A.5) would be zero in the absence of the subleading poly-instanton contribution.

It is straightforward, but long, to extract the eigenvalues and the eigenvectors of the mass matrix of eq. (A.5), working at leading order in an expansion in inverse powers of the volume. The expressions of the eigenvectors turn out to be particularly complicated. It is convenient to present them at leading order in an expansion in the quantity $\tau_{s}$, that we can assume to be small with respect to the other quantities. We find:

$$
\mathcal{C}=\left(\begin{array}{ccc}
\frac{2 \tau_{f}}{\sqrt{3}} & \sqrt{\frac{2}{3}} \tau_{f} & 0 \\
\frac{c_{0}}{\mathcal{V}^{p-1}} & \sqrt{\frac{3}{2}} \mathcal{V} & -\tau_{s}^{3 / 4} \sqrt{3 \mathcal{V}} \\
\frac{c_{1}}{\mathcal{V}^{p}} & \sqrt{6} \tau_{s} & \frac{2 \tau_{s}{ }^{1 / 4} \sqrt{\mathcal{V}}}{\sqrt{3}}
\end{array}\right)
$$

The two last entries in the first column are suppressed by powers of the volume, and are due to the effect of the poly-instanton corrections. The quantities $c_{0}$ and $c_{1}$ are independent from the volume (at leading order in a inverse volume expansion) and read:

$$
\begin{aligned}
& c_{0}=-\frac{\tau_{s}^{\frac{p}{2}-1}}{2 e \pi W_{0}} 3^{-\frac{3}{2}+p}\left(-2 a A C_{1} \pi+b B\left(c\left(-C_{1}+C_{2}\right)+2 C_{1} \pi\right)\right)\left(\frac{W_{0}}{b B-a A}\right)^{1+p}, \\
& c_{1}=\frac{4 \tau_{s}^{\frac{p}{2}} 3^{-\frac{3}{2}+p}\left(2 a A C_{1} \pi+b B\left(c\left(C_{1}-C_{2}\right)-2 C_{1} \pi\right)\right)\left(\frac{W_{0}}{-a A+b B}\right)^{p}}{(a A-b B) e \pi} .
\end{aligned}
$$

The lightest eigenvalue of the mass matrix scales as $m_{\phi}^{2} \propto 1 / \mathcal{V}^{3+p}$, and provides the effective mass for the quintessence field in the poly-instanton dominated region of the quintessence potential (region II of figure 1).

In this region, the volume and $\tau_{s}$ are stabilised at their minima since their mass is very large. From eqs (A.3) and (A.6), one obtains that the displacement of the light field $\tau_{f}$ from its minimum can be expressed in terms of its canonical counterpart as:

$$
\delta \tau_{f} \simeq \frac{2}{\sqrt{3}} \tau_{f} \delta \phi \quad \Rightarrow \quad \tau_{f} \simeq e^{\frac{2}{\sqrt{3}} \phi},
$$

justifying the canonical normalisation we used in eq. (3.8). The diagonalising matrix (A.6), when compared with (A.3), also tells us that, within the approximations we are using, we 
can write the modulus $\tau_{s}$ in terms of canonically normalised fields as:

$$
\frac{\delta \tau_{s}}{\left\langle\tau_{s}\right\rangle} \simeq \frac{c_{1}}{\mathcal{V}^{p}} \delta \phi+\sqrt{6} \delta \chi_{\mathcal{V}}+\frac{2 \sqrt{\mathcal{V}}}{\sqrt{3}\left\langle\tau_{s}\right\rangle^{3 / 4}} \delta \chi_{s},
$$

reproducing the scalings of eq. (4.1).

\section{B Explicit form of the coefficients of the mass matrix}

Here we collect the complete expressions for the quantities $f_{i}$ appearing in the mass matrix $M_{i j}$, eq. (A.5) (we use the same conventions introduced in our section 3):

$$
\begin{aligned}
f_{1}= & \frac{\left((a-b) b B \tau_{s}+Z-a \tau_{s} Z\right)}{\left(4(a-b) b B \tau_{s}+Z-4 a \tau_{s} Z\right)} \\
f_{2}= & \frac{3 \sqrt{\tau_{s}} W_{0}\left(b^{2} B \tau_{3}-Z+a \tau_{s}(-b B+Z)\right)}{Z\left(4 b^{2} B \tau_{s}-Z+4 a \tau_{s}(-b B+Z)\right)} \\
f_{3}= & \frac{8}{3}\left(-36\left(-1+f_{1}\right) f_{1} \tau_{s} W_{0}^{2}-9 f_{2} \sqrt{\tau_{s}} W_{0} Z+2 f_{2}^{2} Z^{2}\right) \\
f_{4}= & \frac{4}{3} f_{2}\left(-f_{2} Z\left(4(a-b) b B \tau_{s}+Z-4 a \tau_{s} Z\right)+6 \sqrt{\tau_{s}} W_{0}\left((a-b) b B \tau_{s}+Z-a \tau_{s} Z\right)\right) \\
f_{5}= & \frac{2}{3} f_{2}\left[-6 \tau_{s}^{3 / 2} W_{0}\left(b(-a+b) B\left(-2+(a+b) \tau_{s}\right)+a\left(-2+a \tau_{s}\right) Z\right)+f_{2}\left(8(a-b)^{2} b^{2} B^{2} \tau_{s}^{2}\right.\right. \\
& \left.\left.+8 b(-a+b) B \tau_{s}\left(-1+3 a \tau_{s}+b \tau_{s}\right) Z+\left(-1+8 a \tau_{s}\left(-1+2 a \tau_{s}\right)\right) Z^{2}\right)\right] \\
f_{6}= & -\frac{16 \pi f_{2}{ }^{1+p}}{3 e}\left[3 b B\left(-c\left(C_{1}-C_{2}\right)\left(-1+\pi \tau_{f}\right)+\left(-a C_{1}+b C_{2}\right) \pi \tau_{s}\right) W_{0}\right. \\
& \left.+\pi\left(4 B\left(a C_{1}+b\left((-1+b) C_{1}-b C_{2}\right)\right) f_{2} \sqrt{\tau_{s}}+3 C_{1}\left(-2+2 \pi \tau_{f}+a \tau_{s}\right) W_{0}\right) Z-4 a C_{1} f_{2} \pi \sqrt{\tau_{s}} Z^{2}\right] \\
f_{7}= & \frac{4 f_{2}{ }^{1+p}}{3 e \sqrt{\tau_{s}}}\left[3 b B \sqrt{\tau_{s}}\left(-2 a C_{1} \pi-2 b^{2} C_{2} \pi \tau_{s}+b\left(c\left(C_{1}-C_{2}\right)\left(-1+2 \pi \tau_{f}\right)+2 \pi\left(C_{2}+a C_{1} \tau_{s}\right)\right)\right) W_{0}\right. \\
& -2 \pi\left(2 B\left(a C_{1}+b\left((-1+b) C_{1}-b C_{2}\right)\right) f_{2}\left(-1+4 b \tau_{s}\right)+3 C_{1} \sqrt{\tau_{s}}\left(b\left(-1+2 \pi \tau_{f}\right)+a\left(-1+b \tau_{s}\right)\right) W_{0}\right) Z \\
& \left.+4 a C_{1} f_{2} \pi\left(-1+4 b \tau_{s}\right) Z^{2}\right] \\
f_{8}= & \frac{8 f_{2}{ }^{1+p}}{3 e}\left[3 b B\left(c\left(C_{1}-C_{2}\right)\left(-1+2 \pi \tau_{f}\right)+2\left(a C_{1}-b C_{2}\right) \pi \tau_{s}\right) W_{0}\right. \\
& \left.-2 \pi\left(2 B\left(a C_{1}+b\left((-1+b) C_{1}-b C_{2}\right)\right) f_{2} \sqrt{\tau_{s}}+3 C_{1}\left(-1+2 \pi \tau_{f}+a \tau_{s}\right) W_{0}\right) Z+4 a C_{1} f_{2} \pi \sqrt{\tau_{s}} Z^{2}\right]
\end{aligned}
$$

\section{References}

[1] E. J. Copeland, M. Sami and S. Tsujikawa, Int. J. Mod. Phys. D 15 (2006) 1753 [hep-th/0603057].

[2] C. F. Kolda and D. H. Lyth, Phys. Lett. B 458 (1999) 197 [hep-ph/9811375].

[3] E. G. Adelberger, B. R. Heckel and A. E. Nelson, Ann. Rev. Nucl. Part. Sci. 53 (2003) 77 [arXiv:hep-ph/0307284].

[4] S. M. Carroll, Phys. Rev. Lett. 81 (1998) 3067 [astro-ph/9806099].

[5] K. Choi, Phys. Rev. D 62 (2000) 043509 [hep-ph/9902292]. 
[6] N. Kaloper and L. Sorbo, Phys. Rev. D 79 (2009) 043528 [arXiv:0810.5346 [hep-th]].

[7] S. Panda, Y. Sumitomo and S. P. Trivedi, Phys. Rev. D 83 (2011) 083506 [arXiv:1011.5877 [hep-th]].

[8] G. Gupta, S. Panda and A. A. Sen, Phys. Rev. D 85 (2012) 023501 [arXiv:1108.1322 [astro-ph.CO]].

[9] M. Cicoli, F. G. Pedro and G. Tasinato, JCAP 1112 (2011) 022 [arXiv:1110.6182 [hep-th]].

[10] M. Cicoli, C. P. Burgess and F. Quevedo, JHEP 1110 (2011) 119 [arXiv:1105.2107 [hep-th]].

[11] Y. Aghababaie, C. P. Burgess, S. L. Parameswaran and F. Quevedo, Nucl. Phys. B 680 (2004) 389 [arXiv:hep-th/0304256];

Y. Aghababaie et al., JHEP 0309 (2003) 037 [arXiv:hep-th/0308064];

J. Vinet, J. M. Cline, Phys. Rev. D71 (2005) 064011 [hep-th/0501098].

[12] A. Albrecht, C. P. Burgess, F. Ravndal and C. Skordis, Phys. Rev. D 65 (2002) 123507 [astro-ph/0107573].

[13] C. P. Burgess, AIP Conf. Proc. 743 (2005) 417 [hep-th/0411140].

[14] H. -P. Nilles, A. Papazoglou and G. Tasinato, Nucl. Phys. B 677 (2004) 405 [hep-th/0309042].

[15] C. P. Burgess and L. van Nierop, JHEP 1104 (2011) 078 [arXiv:1101.0152 [hep-th]].

[16] M. Cicoli, M. Kreuzer and C. Mayrhofer, JHEP 1202 (2012) 002 [arXiv:1107.0383 [hep-th]].

[17] V. Balasubramanian, P. Berglund, J. P. Conlon and F. Quevedo, JHEP 0503 (2005) 007 [arXiv:hep-th/0502058].

[18] R. Blumenhagen, S. Moster and E. Plauschinn, JHEP 0801 (2008) 058 [arXiv:0711.3389 [hep-th]].

[19] M. Cicoli, C. Mayrhofer and R. Valandro, JHEP 1202 (2012) 062 [arXiv:1110.3333 [hep-th]].

[20] S. Kachru, R. Kallosh, A. D. Linde and S. P. Trivedi, Phys. Rev. D 68 (2003) 046005 [hep-th/0301240].

[21] C. P. Burgess, R. Kallosh and F. Quevedo, JHEP 0310 (2003) 056 [hep-th/0309187].

[22] M. Cicoli, A. Maharana, F. Quevedo and C. P. Burgess, arXiv:1203.1750 [hep-th].

[23] M. Cicoli, J. P. Conlon and F. Quevedo, JHEP 0801 (2008) 052 [arXiv:0708.1873 [hep-th]]. M. Cicoli, J. P. Conlon and F. Quevedo, JHEP 0810 (2008) 105 [arXiv:0805.1029 [hep-th]].

[24] R. Blumenhagen and M. Schmidt-Sommerfeld, JHEP 0807 (2008) 027 [arXiv:0803.1562 [hep-th]].

[25] J. P. Conlon, R. Kallosh, A. D. Linde and F. Quevedo, JCAP 0809 (2008) 011 [arXiv:0806.0809 [hep-th]].

[26] M. Cicoli, G. Tasinato, I. Zavala, C. P. Burgess and F. Quevedo, arXiv:1202.4580 [hep-th].

[27] J. P. Conlon, D. Cremades and F. Quevedo, JHEP 0701 (2007) 022 [arXiv:hep-th/0609180].

[28] E. J. Copeland, A. R. Liddle and D. Wands, Phys. Rev. D 57 (1998) 4686 [arXiv:gr-qc/9711068].

[29] S. Hellerman, N. Kaloper and L. Susskind, JHEP 0106 (2001) 003 [hep-th/0104180].

[30] W. Fischler, A. Kashani-Poor, R. McNees and S. Paban, JHEP 0107 (2001) 003 [arXiv:hep-th/0104181].

[31] L. Amendola, Phys. Rev. D 62 (2000) 043511 [astro-ph/9908023].

[32] C. G. Boehmer, G. Caldera-Cabral, R. Lazkoz and R. Maartens, Phys. Rev. D 78 (2008) 023505 [arXiv:0801.1565 [gr-qc]]. 
[33] J. P. Conlon and F. Quevedo, JCAP 0708 (2007) 019 [arXiv:0705.3460 [hep-ph]].

[34] M. Cicoli and A. Mazumdar, JCAP 1009 (2010) 025 [arXiv:1005.5076 [hep-th]].

[35] L. Anguelova, V. Calo and M. Cicoli, JCAP 0910 (2009) 025 [arXiv:0904.0051 [hep-th]]. 